\title{
Inhibition of interferon response by cystatin B: implication in HIV replication of macrophage reservoirs
}

\author{
L. Rivera-Rivera $\cdot$ J. Perez-Laspiur • Krystal Colón • \\ L. M. Meléndez
}

Received: 19 July 2011 /Revised: 4 November 2011 /Accepted: 8 November 2011 /Published online: 7 December 2011

(C) The Author(s) 2011. This article is published with open access at Springerlink.com

\begin{abstract}
Cystatin B and signal transducer and activator of transcription-1 (STAT-1) phosphorylation have recently been shown to increase human immunodeficiency virus1 (HIV-1) replication in monocyte-derived macrophages (MDM), but the molecular pathways by which they do are unknown. We hypothesized that cystatin B inhibits the interferon (IFN) response and regulates STAT-1 phosphorylation by interacting with additional proteins. To test if cystatin $\mathrm{B}$ inhibits the IFN- $\beta$ response, we performed luciferase reporter gene assays in Vero cells, which are IFN deficient. Interferon-stimulated response element (ISRE)-driven expression of firefly luciferase was significantly inhibited in Vero cells transfected with a cystatin B expression vector compared to cells transfected with an empty vector. To determine whether cystatin B interacts with other key players regulating STAT-1 phosphorylation and HIV-1 replication, cystatin B was immunoprecipitated from HIV-1-infected MDM. The protein complex was analyzed by liquid chromatography tandem mass spectrometry. Protein interactions with cystatin $\mathrm{B}$ were verified by Western blots and immunofluorescence with confocal imaging. Our findings confirmed that cystatin $\mathrm{B}$ interacts with pyruvate kinase M2 isoform, a protein previously associated cocaine enhancement of HIV-1 replication, and major
\end{abstract}

L. Rivera-Rivera $\cdot K$. Colón $\cdot$ L. M. Meléndez $(\triangle)$

Department of Microbiology and NeuroAIDS Program,

University of Puerto Rico-Medical Sciences Campus,

San Juan, Puerto Rico 00935

e-mail: loyda.melendez@upr.edu

J. Perez-Laspiur

RCMI Translational Proteomics Center,

University of Puerto Rico-Medical Sciences Campus,

San Juan, Puerto Rico 00935 vault protein (MVP), an IFN-responsive protein that interferes with JAK/STAT signals. Western blot studies confirmed the interaction with pyruvate kinase M2 isoform and MVP. Immunofluorescence studies of HIV-1-infected MDM showed that upregulated MVP colocalized with STAT-1. To our knowledge, the current study is the first to demonstrate the coexpression of cystatin B, STAT-1, MVP, and pyruvate kinase M2 isoform with HIV-1 replication in MDM and thus suggests novel targets for HIV-1 restriction in macrophages, the principal reservoirs for HIV-1 in the central nervous system.

Keywords Cystatin B $\cdot$ HIV-1 replication - STAT-1 signaling · MVP · Monocyte-derived macrophages . Interferon response

$\begin{array}{ll}\text { Abbreviations } \\ \text { CAF } & \text { CD8 T lymphocyte antiviral factor } \\ \text { CNS } & \text { Central nervous system } \\ \text { HIV-1 } & \text { Human immunodeficiency virus-1 } \\ \text { IFN } & \text { Interferon } \\ \text { IRF-1 } & \text { Interferon regulatory factor-1 } \\ \text { ISG } & \text { Interferon-stimulated gene } \\ \text { LC-MS/MS } & \begin{array}{l}\text { Liquid chromatography tandem mass } \\ \text { spectrometry }\end{array} \\ \text { LTR } & \text { Long terminal repeat } \\ \text { MDM } & \text { Monocyte-derived macrophage } \\ \text { MVP } & \text { Major vault protein } \\ \text { PKM2 } & \text { Pyruvate kinase M2 } \\ \text { STAT-1 } & \text { Signal transducer and activator of } \\ & \text { transcription-1 } \\ \text { STAT-1PS } & \text { STAT-1 serine phosphorylation } \\ \text { STAT-1PY } & \text { Tyrosine-phosphorylated STAT-1 } \\ \text { TFA } & \text { Trifluoroacetic acid }\end{array}$




\section{Introduction}

Monocytes, macrophages, and microglia are mononuclear phagocytes important in innate immunity and also key reservoirs of human immunodeficiency virus-1 (HIV-1) in the central nervous system (CNS). These reservoirs present a challenge to HIV eradication as they continue to produce virus in tissue despite antiretroviral therapy. Our group recently showed that cystatin $\mathrm{B}$, a cysteine protease inhibitor, is upregulated in blood monocyte-derived macrophages (MDM) compared to placental macrophages, which are less susceptible than MDM to HIV-1 infection (Luciano-Montalvo et al. 2008). Intracellular expression of cystatin B was increased in HIV-1-infected MDM at 12 days post-infection (Luciano-Montalvo et al. 2008) and in the secretome of HIV1-infected MDM (Ciborowski et al. 2007; Garcia-Crespo et al. 2009), suggesting that this protein is activated during HIV infection. A direct connection between cystatin B and HIV replication was demonstrated using small interfering RNA against cystatin B (Luciano-Montalvo et al. 2008). Subsequently, the signaling mechanisms for cystatin B in HIV replication were related to its interaction with signal transducer and activator of transcription-1 (STAT-1) (Luciano-Montalvo and Melendez 2009). Whereas STAT-1 activates HIV-1 replication, high levels of tyrosine phosphorylated STAT-1 (STAT-1PY) have been associated with HIV-1 inhibitory activity (Chang et al. 2002). Interestingly, recent data from our laboratory using more specific monoclonal antibodies showed higher levels of STAT-1PY in placental macrophages, a cell that restricts HIV replication, than in MDM (Luciano-Montalvo and Melendez 2009), and very low levels in MDM until 12 days after infection. Low levels of cystatin B together with high levels of STAT1PY may restrict HIV replication in placental macrophages, whereas high levels of cystatin B and low levels of STAT1PY may promote HIV replication in MDM (LucianoMontalvo and Melendez 2009). However, the signaling pathways and mechanisms for increased HIV-1 replication in MDM are yet to be determined.

Although cystatin B is recognized primarily as a cysteine protease inhibitor rather than a regulatory protein, Di Giamo et al. identified five recombinant proteins that interact with cystatin B, none of which is a protease (Di Giamo et al. 2002). Those investigators suggested a role of the cystatin B multiprotein complex in the cerebellum; disruption of which correlated with the etiology and pathogenesis of progressive myoclonus epilepsy, a degenerative disease of the central nervous system. That was the first time cystatin B was reported to be part of a multiprotein complex with an unknown function. In the present study, we identified proteins interacting with cystatin B in order to elucidate the relationship between this protein, STAT-1 phosphorylation, and HIV-1 replication in MDM.
Type I interferons $\alpha$ and $\beta$ (IFN- $\alpha$ and IFN- $\beta$ ) are antiviral proteins important in innate immunity. The production of IFN- $\beta$ is a rapid and very effective antiviral response to HIV in cultured macrophages (Gessani et al. 1994a, b). In the present study, we found that cystatin B inhibited the IFN- $\beta$ response in Vero cells by preventing STAT-1 translocation to the nucleus and decreasing levels of STAT-1PY. The discovery of this mechanism of cystatin B regulation of STAT-1 phosphorylation could inform the development of new therapeutic approaches that aim to inhibit the long terminal repeat (LTR)-mediated HIV replication by modulating the site of STAT-1 phosphorylation in favor of tyrosine instead inhibiting STAT-1 expression.

\section{Results}

Proteins associated with cystatin $B$ To determine whether cystatin B interacts with other key players regulating STAT1 phosphorylation and HIV-1 replication, cystatin B was immunoprecipitated from HIV-1-infected MDM as described previously (Luciano-Montalvo and Melendez 2009), and the protein complex was analyzed by liquid chromatography tandem mass spectrometry (LC-MS/ MS). We found that cystatin B interacts with many different proteins in HIV-1-infected cells, including regulatory, glycolytic, metabolic, structural, transport, proteases, and immune proteins (Table 1). Most of the proteins identified ( 25 of 37 ) associated with cystatin B in HIV-1-infected cells but not in uninfected controls. Two proteins did not associate with cystatin B in HIV-infected cells, but did so in uninfected controls: cathepsin B (a cysteine protease inhibited by cystatin B) and elongation factor 1 . Bound proteins were not detected in the negative control, pre-cleared MDM lysates incubated with unconjugated beads.

Validation of immunoprecipitated proteins Major vault protein (MVP), and pyruvate kinase M2 (PKM2) were selected for validation by Western blot after coimmunoprecipitation with cystatin $\mathrm{B}$ antibody because these proteins are known to be involved in the regulation of STAT-1 phosphorylation and HIV-1 replication. Western blot studies confirmed that cystatin B interacted with PKM2 isoform, a protein previously associated with cocaine enhancement of HIV-1 replication, and MVP, an IFN-responsive protein that interferes with IFN-activated JAK/STAT signals (Fig. 1).

Studies of HIV-infected and uninfected MDM using immunofluorescence and confocal imaging demonstrated overexpression of MVP in HIV-infected cells (Fig. 2). These results extend those of Steiner et al. (2006), who 
Table 1 Cystatin B-associated proteins from HIV-1-infected MDM and uninfected controls

\begin{tabular}{|c|c|c|c|c|}
\hline \multirow[t]{2}{*}{ Protein name } & \multirow{2}{*}{$\begin{array}{l}\text { Accession } \\
\text { UniProt }\end{array}$} & \multirow{2}{*}{$\begin{array}{l}\text { Molecular } \\
\text { Weight (Da) }\end{array}$} & \multicolumn{2}{|c|}{ \# peptide } \\
\hline & & & Control & $\mathrm{HIV}+$ \\
\hline \multicolumn{5}{|l|}{ Regulatory proteins } \\
\hline Major vault protein & Q14764 & $99,326.3$ & 3 & 14 \\
\hline Lymphocyte-specific protein 1 & P33241 & $37,191.3$ & 0 & 2 \\
\hline \multicolumn{5}{|l|}{ Glycolytic enzymes } \\
\hline Pyruvate kinase M1/M2 & P14618 & $57,936.6$ & 4 & 6 \\
\hline Human alpha-enolase & P06733 & $47,168.7$ & 0 & 6 \\
\hline Glyceraldehyde-3 phosphate dehydrogenase & P04406 & $36,053.0$ & 0 & 5 \\
\hline L-lactate dehydrogenase A & P00338 & $36,688.5$ & 0 & 2 \\
\hline Fructose-1,6-bisphosphatase 1 & P09467 & $36,814.2$ & 0 & 4 \\
\hline \multicolumn{5}{|l|}{ Metabolic proteins } \\
\hline Peroxisomal multifunctional enzyme type 2 & P51659 & $79,685.9$ & 0 & 2 \\
\hline Sulfide: quinoneoxidoreductasemitochondrial & Q9Y6N5 & $49,960.4$ & 0 & 5 \\
\hline Transketolase & P29401 & 65,333 & 0 & 2 \\
\hline Heat shock protein HSP90 alpha & P07900 & $84,659.1$ & 0 & 2 \\
\hline V-type proton ATPase subunit B, brain isoform & P21281 & $56,500.4$ & 0 & 2 \\
\hline ATP synthase subunit alpha, mitochondrial & P25705 & $59,750.3$ & 0 & 3 \\
\hline Synaptic vesicle membrane protein VAT-1 homolog & Q99536 & 41,920 & 0 & 3 \\
\hline \multicolumn{5}{|l|}{ Structural proteins } \\
\hline Elongation factor 1 & P68104 & $50,140.5$ & 2 & 0 \\
\hline Annexin A2 & P07355 & $38,603.8$ & 4 & 8 \\
\hline Myosin 9 (MYH9) & P35579 & $226,529.8$ & 5 & 36 \\
\hline Actin Cytoplasmic 1/2 & P63261 & $41,736.5$ & 5 & 9 \\
\hline Vimentin & P08670 & $53,651.3$ & 2 & 9 \\
\hline Macrophage-capping protein & P40121 & $38,517.3$ & 2 & 5 \\
\hline Cofilin-1 & P23528 & $18,502.4$ & 0 & 2 \\
\hline Plastin-2 & P13796 & $70,288.9$ & 4 & 11 \\
\hline Myosin regulatory light chain & P05976 & 21,145 & 0 & 4 \\
\hline Moesin & P26038 & $67,819.5$ & 0 & 4 \\
\hline Protein disulfideisomeraseA6 & Q15084 & 48,121 & 6 & 2 \\
\hline Human Filamin-A & P21333 & $280,735.4$ & 0 & 6 \\
\hline Gelsolin & P06396 & $85,696.9$ & 0 & 3 \\
\hline Ras GTPase- activating-like protein & P46940 & 189,252 & 0 & 8 \\
\hline Alpha-actinin-1 & P12814 & $103,056.9$ & 0 & 2 \\
\hline Protein S100-A11 & P31949 & $11,740.4$ & 0 & 2 \\
\hline \multicolumn{5}{|l|}{ Transport-related proteins } \\
\hline Clathrin heavy chain 1 & Q00610 & $191,612.6$ & 6 & 3 \\
\hline ADP-ribosylation factor $1 / 3$ & Q9NZ52 & $20,696.6$ & 0 & 2 \\
\hline Transferrin receptor protein 1 & Q5RDH6 & 84,871 & 0 & 2 \\
\hline \multicolumn{5}{|l|}{ Proteases } \\
\hline Cathepsin B & P07858 & 37,822 & 2 & 0 \\
\hline Cathepsin D & P07339 & 44,552 & 0 & 2 \\
\hline \multicolumn{5}{|l|}{ Immune protein } \\
\hline HLA class I histocompatibility antigen & P30488 & 45,000 & 0 & 2 \\
\hline
\end{tabular}

previously reported MVP-STAT coexpression in tumor cells induced by IFN- $\gamma$. To our knowledge, the current study is the first to demonstrate the coexpression of STAT-1 and MVP in HIV-1-infected MDM.
Cystatin B inhibition of IFN- $\beta$ response in Vero cells To test if cystatin $B$ inhibits the IFN- $\beta$ response, we performed luciferase reporter gene assays in Vero cells, which are IFN deficient. Robust IFN stimulation of gene 54 (ISG54)- 


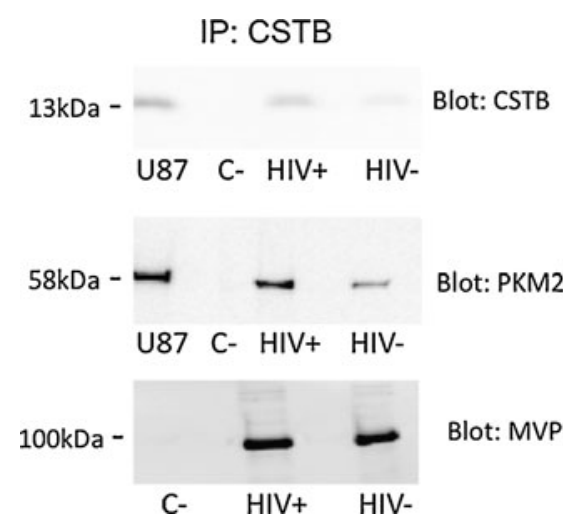

Fig. 1 Validation of immunoprecipitated proteins by Western blot. MVP, and PKM2 were selected to be validated by Western blot after co-immunoprecipitation with cystatin B antibody because they are involved in the regulation of STAT-1 phosphorylation and HIV-1 replication. CSTB cystatin B; U87 U87 whole cell lysates used as positive controls; $C$ - controls for non-specific binding using unconjugated beads and MDM lysates; HIV-1 + MDM lysates inoculated with HIV-1 ${ }_{\mathrm{ADA}}, H I V-1$ uninfected MDM lysates

driven expression of firefly luciferase was seen in cells transfected with an empty vector, and this stimulation was abolished in cells transfected with a cystatin B vector (Fig. 3). Furthermore, the interferon response of IFN- $\beta$-treated Vero cells expressing cystatin B was significantly inhibited as compared with the results before IFN treatment.

Inhibition of STAT-1 nuclear translocation and tyrosine phosphorylation in response to cystatin $B$ We used immunofluorescence and confocal imaging on Vero cells transiently transfected with a vector expressing cystatin $\mathrm{B}$ to determine if this protein alters nuclear translocation of STAT-1. Vero cells transfected with an empty vector or a vector expressing NIPAH-V were used as negative and positive controls, respectively. NIPAH-V was used because it is known to inhibit the IFN- $\beta$ response via inhibition of STAT-1 translocation to the nucleus (Rodriguez et al. 2002).
Before IFN- $\beta$ treatment, all STAT-1 immunoreactivity was localized to the cytosol. STAT-1 immunoreactivity was translocated to the nucleus after IFN- $\beta$ treatment in the negative empty control, but retained in the cytoplasm of the NIPAH-V control, and in Vero cells expressing cystatin B (Fig. 4).

The effect of cystatin B on the expression of STAT-1PY was determined by Western blots (Fig. 5). Vero cells were transfected with cystatin B plasmid for a period of $24 \mathrm{~h}$. Overexpression of cystatin B was demonstrated in transfected cells with cystatin B vector after normalization with glyceraldehyde 3-phosphate dehydrogenase (GAPDH) $\left({ }^{*} p<\right.$ 0.05) (Fig. 5a, b). Cystatin B inhibited STAT-PY in Vero cells $\left({ }^{*} p<0.05\right)$ (Fig. 5a, c). When IFN was added to cultures in presence of cystatin $B$, there was a reduction in STAT-PY relative to cystatin B-untreated cells that approached significance ( $p=0.06$ ) (Fig. 5a, c). These results demonstrated that cystatin $\mathrm{B}$ inhibits the IFN- $\beta$ response and the nuclear translocation of STAT-1 and a tendency to decreases the expression of STAT-1PY in Vero cells.

\section{Discussion}

HIV-1-associated neurocognitive disorders result from HIV1 infection of the brain, alterations in the blood-brain barrier, migration of activated monocytes, and a subsequent induced immune activation response. HIV-1 replication and neurotoxicity are both related to the type of STAT-1 phosphorylation. STAT-1 serine phosphorylation (STAT1PS) promotes blood-brain barrier damage (Chaudhuri et al. 2008), whereas STAT-1PY has been associated with HIV-1 inhibitory activity (Chang et al. 2002). Cystatin B has been associated with decreased levels of STAT-1PY and increased HIV-1 replication in MDM (Luciano-Montalvo and Melendez 2009). However, the pathways and the
Fig. 2 Overexpression of MVP in HIV-1-infected MDM at 12 days post-infection. Immunofluorescence and confocal imaging confirmed that MVPSTAT coexpression is increased in HIV-1-infected MDM. Control uninfected MDM; HIV-1 + HIV-1-infected MDM at 12 days post-infection. Green fluorescence represents MVP and red fluorescence represents STAT-1
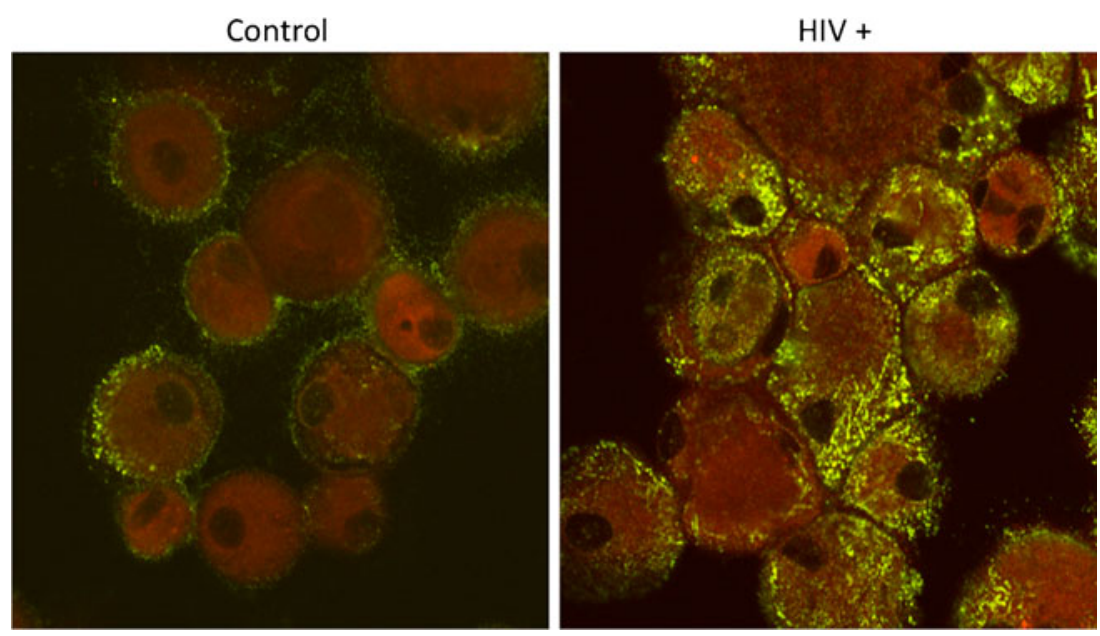
A.

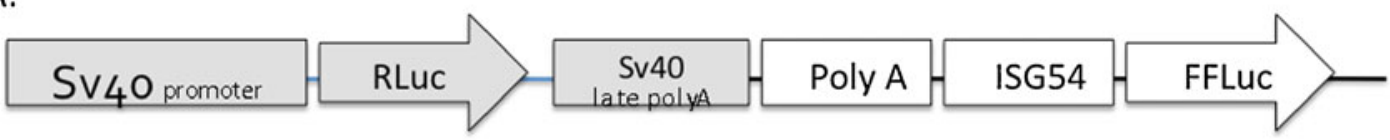

B.

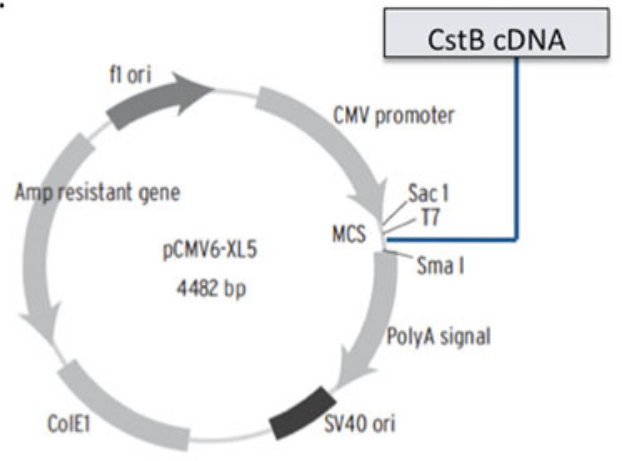

C.

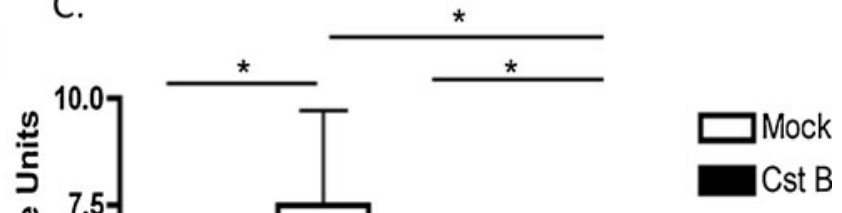

Fig. 3 Cystatin B inhibition of IFN- $\beta$ response in Vero cells. Interferon stimulation of gene 54 (ISG54)-driven expression of firefly luciferase was significantly inhibited in cells transfected with a cystatin B vector compared to cells transfected with the empty vector. a Plasmid construct containing the ISG54 promoter fused to firefly luciferase and the SV40 promoter fused to Renilla luciferase. b Vector used to induce expression of cystatin B. c Luciferase reporter gene assays in Vero cells. Cells were

mechanisms by which cystatin B interacts with STAT-1 are unknown. Recently, cystatin B has also been shown to interact with cathepsin B in HIV-negative macrophages but fails to interact in HIV-negative MDM (Rodriguez et al. 2010). More recently, our group found that cystatin B also fails in protecting against HIV-1 neuropathogenesis by lack of inhibition of the cysteine protease cathepsin B, a potent neurotoxin. In this study, we provide evidence that cystatin $B$ interacts with many different proteins in HIV-1-infected MDM, indicating that cystatin B performs other functions in addition to inhibiting cysteine protease. Furthermore, our results demonstrated that cystatin B inhibits the IFN- $\beta$ response and inhibits the nuclear translocation of STAT-1 and demonstrated a tendency to decreases the expression of STAT-1PY in Vero cells.

Our findings support the hypothesis that cystatin B interacts with proteins related to HIV-1 replication. Five enzymes associated with the glycolytic pathway, including the PKM2 isoform, were identified as cystatin B-associated proteins. PKM2 has been reported to be upregulated in association with cocaine enhancement of HIV-1 replication in normal human astrocytes (Reynolds et al. 2006). In contrast, PKM2 is downregulated in HIV-1/vesicular stomatitis virus-infected murine microglia (Wang et al. 2008). This downregulation of PKM2 occurs in parallel with the low levels of cystatin B associated with HIV-1 restriction in microglia (manuscript in revision). Cystatin B also seems to interact with proteins transiently transfected with an empty vector (mock) or a vector expressing cystatin B $(C s t B)$ and stimulated with $500 \mathrm{U} / \mathrm{mL}$ IFN $\left(I F N^{+}\right)$or left untreated $\left(I F N^{-}\right)$. Twenty-four hours posttreatment, the cells were lysed and the luciferase activity was measured by using a dual luciferase assay (Promega). Firefly luciferase units were divided by Renilla units to obtain relative luciferase units (RLU). Mean of four independent experiments, ${ }^{*} p<0.05$. Error bars represent \pm SEM

involved in the regulation of STAT-1 phosphorylation. One of these proteins is MVP, the main component of vaults. Vaults are large ribonucleoprotein particles implicated in the regulation of cellular signaling cascades and may act as scaffolds for proteins involved in signal transduction. The vaults are composed of three proteins: MVP, the telomeraseassociated protein and the vault poly (ADP-ribose) polymerase. MVP has multiple roles in signal pathway regulation and immunity (Berger et al. 2009) and is associated with PTEN and PI3K (Yu et al. 2002), ERK and EGFR/SHP2/MAPK/Src (Kolli et al. 2004), multidrug resistance (Kitazono et al. 1999, 2001), and proteins associated with the ubiquitin pathway as COP1 (Yi et al. 2005). In addition to all these functions, MVP is an IFN- $\gamma$-responsive gene that interferes with the IFN- $\gamma$-induced antiviral response mediated by STAT-1 signaling (Steiner et al. 2006). Using tandem mass spectrometry, we found that MVP is also associated with cystatin B. In the present study, STAT-1 was not detected among the proteins associated with cystatin B by mass spectrometry. However, the co-immunoprecipitation of cystatin B and STAT-1 in HIV-infected MDM was previously demonstrated by our group using direct Western blot detection of STAT-1 in the cystatin B-immunoprecipitated pool of proteins (LucianoMontalvo and Melendez 2009). A possible explanation for the absence of STAT-1 in the IP LC-MS/MS analysis could be poor ionization of these peptides, masking by other ionized peptides, or data that did not pass our conservative 


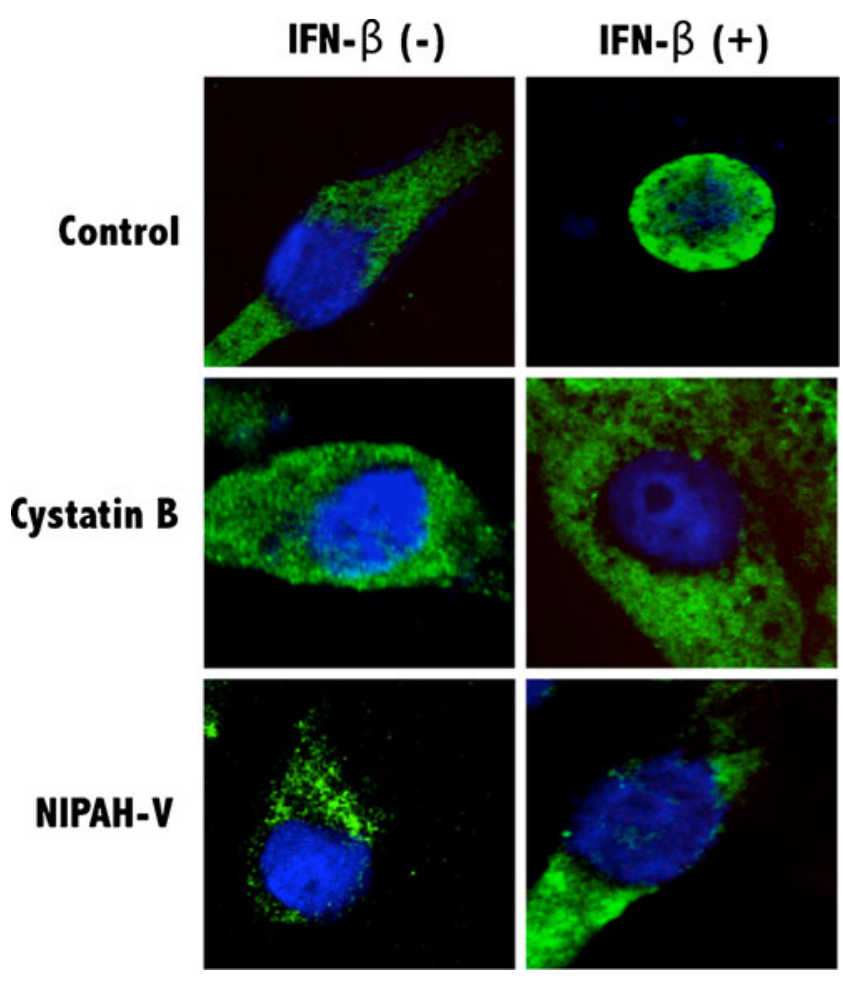

Fig. 4 Inhibition of nuclear translocation of STAT-1 by cystatin B expression. Immunofluorescence and confocal imaging of Vero Cells transiently transfected with a vector expressing cystatin B shows that cystatin B alters the nuclear translocation of STAT-1 after treatment with $500 \mathrm{U} / \mathrm{mL}$ IFN- $\beta$. Vero cells transfected with an empty vector (control) or a vector expressing NIPAH-V were used as negative and positive controls, respectively. Blue fluorescent DAPI nucleic acid stain was used as nuclear stain

exclusion criteria. The LC-MS/MS data are conservatively analyzed to accept as positive identifications only those data that pass these exclusion criteria: BioWorks ${ }^{\circledR}$ unified score of $>3,000$, Xcorr for doubly charged precursor ion of $>2.5$, DeltaCn of $>0.3$, more than $60 \%$ of fragment ions per sequenced peptide, and at least two peptides per protein for identification.

Interestingly, the other cystatin B-associated protein related to HIV-1 replication, PKM2, is co-regulated at the gene and protein level by STAT-1 (Pitroda et al. 2009) and is a phosphotyrosine-binding protein that binds directly and selectively to tyrosine-phosphorylated peptides (Christofk et al. 2008). In the present study, the MVP and PKM2 proteins were chosen for verification by Western blot because they have been reported in the literature as being involved in HIV1 replication and the regulation of STAT-1 phosphorylation. The presence of both protein bands was confirmed in Western blots of HIV-infected MDM. Furthermore, immunofluoresence and confocal imaging demonstrated increased the expression of MVP that colocalized with STAT-1 in HIV1-infected MDM, as was reported earlier for tumor cells (Steiner et al. 2006). To our knowledge, this is the first study to demonstrate the coexpression of cystatin B and MVP with HIV-1 replication in MDM. This finding points to these proteins as potential novel targets for anti-HIV therapy in macrophages, the principal reservoirs for HIV-1 in the CNS. Future studies will elucidate the exact role that cystatin B interactions with MVP and PKM2 play in STAT-1 phosphorylation and HIV replication in MDM.

Both neurotoxicity and HIV replication are related to the type of STAT-1 phosphorylation. STAT-1PS is detrimental in that it promotes blood-brain barrier damage (Chaudhuri et al. 2008), whereas STAT-1PY is beneficial as it has been associated with HIV-1 inhibitory activity mediated by CD8 T lymphocyte antiviral factor (CAF) (Chang et al. 2002). CAF inhibits LTR-mediated HIV replication by inducing the expression of interferon regulatory factor 1 (IRF-1), which is STAT-1PY dependent. STAT-1 inhibition has been suggested as a new therapeutic approach to clear HIV-1 macrophage reservoirs (Magnani et al. 2003). Taking these data together, we propose that preventing viral replication in macrophages might be accomplished by modulating the site of STAT-1 phosphorylation in favor of tyrosine, as it is known that STAT-1PY inhibits LTR-mediated HIV replication. We also postulate that cystatin B may inhibit the signaling pathway mediated by IFN- $\beta$ by regulating STAT1 tyrosine phosphorylation. This action would prevent the expression of IFN-dependent antiviral genes and promote HIV replication mediated by LTR and IRF-1. To test if cystatin $\mathrm{B}$ inhibits the IFN- $\beta$ response, we performed luciferase reporter gene assays in Vero cells, which are IFN deficient. ISG54-driven expression of firefly luciferase was significantly inhibited in Vero cells expressing cystatin B relative to cells transfected with the empty vector (Fig. 3). These findings allow us to propose two mechanisms for the high levels of HIV replication in MDM mediated by cystatin B: (1) a decrease in IFN- $\beta$-induced antiviral gene expression mediated by the STAT-1/IRF-1 complex and (2) an induction of HIV replication mediated by the NF-K $\beta$ / IRF-1 complex.

Following cellular activation, the binding of cellular transcription factors such as NF- $\mathrm{k} \beta$ to response elements in the HIV-1 promoter results in marked upregulation of HIV replication (Kinoshita et al. 1997). NF-k $\beta$ transcriptional activity requires the IRF-1 to induce HIV replication by formation of a complex at the HIV-1 LTR. The NF-k $\beta /$ IRF-1 complex binds to the gene promoter and induces HIV replication (Sgarbanti et al. 2008). Furthermore, NF-k $\beta$ suppresses both antiviral and immunomodulatory actions of IFN- $\beta$ against influenza virus (Wei et al. 2006), whereas in NF-k $\beta$ knockout mice, IFN- $\beta$ induced the binding of STAT1 and IRF-1 to activate genes (Wei et al. 2006). STAT-1PY has been associated with HIV-1 inhibitory activity (Chang et al. 2002) and recent studies by our group showed that cystatin B is associated with a reduction in STAT-1PY and 
Fig. 5 Decreased levels of STAT-1PY expression are associated with cystatin B expression. The effect of cystatin B on the expression of STAT-1PY was determined by Western blots. Vero cells were either mock transfected or transfected with cystatin B plasmid for a period of $24 \mathrm{~h}$. IFN- $\beta$ (500 U/ $\mathrm{ml}$ ) was added to the cultures. Whole cell lysates were collected after $24 \mathrm{~h}$ of IFN exposure. The expression of STAT-1PY and cystatin $\mathrm{B}$ is shown in presence and absence of IFN (a). Overexpression of cystatin $\mathrm{B}$ is demonstrated in cells transfected with cystatin B vector after normalization with GAPDH $\left({ }^{*} p<0.05\right)($ b). Cells transfected with cystatin B demonstrated a decreased ratio of STAT-1PY over cystatin B and a tendency to decrease in the presence of IFN- $\beta$ ( $p=0.09)$ (c). Control lanes represent cultures that were not transfected with a vector. This experiment is representative of three biological replicates
A.

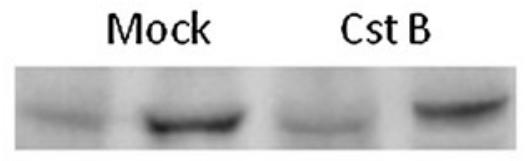

Control
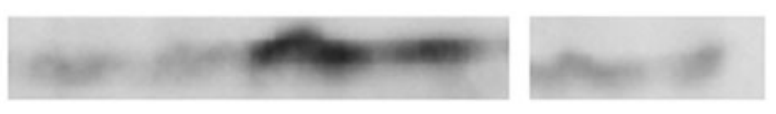

Cst B
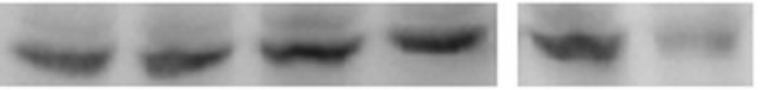

GAPDH
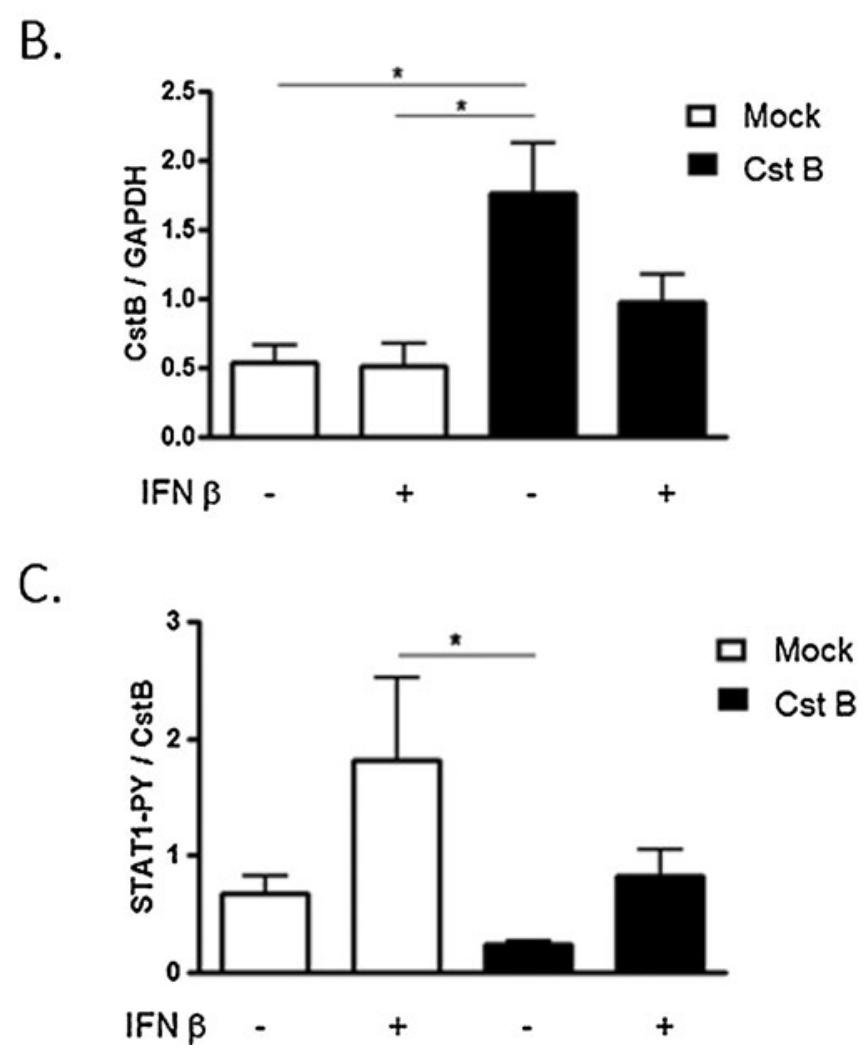

HIV replication in MDM (Luciano-Montalvo and Melendez 2009), and together these findings provide support for a role of cystatin B in HIV replication. On the basis of our results and a review of relevant literature, we propose a mechanism to explain how cystatin B promotes viral replication in macrophage reservoirs (Fig. 6). We propose that the reduction of STAT-1PY prevents the formation of the STAT-1PY/IRF-1 complex, thus inhibiting the expression of antiviral genes induced by IFN- $\beta$. The induction of IRF-1 expression by IFN- $\beta$ (Fujita et al. 1989) and its availability due to low levels of STAT-1PY allows the formation of the NFk- $\beta$ /IRF-1 complex that induces LTR-mediated HIV replication. Future studies will be aimed at elucidating the role of cystatin B in regulating the JAK/STAT-1 pathway and LTR-mediated HIV-1 replication in MDM and determining whether cystatin B is a novel regulatory protein and if MVP plays a role in this interaction. These studies may prove important for the development of inhibitory therapeutic directed against macrophage HIV reservoirs.

\section{Materials and methods}

Monocyte isolation, cultivation, and HIV-1 infection Peripheral blood mononuclear cells were isolated from the peripheral blood of healthy donors after appropriate consents were obtained in accordance with the University of Puerto Rico Medical Sciences Campus Institutional Review Board protocol \#0720109. The cells were seeded into T-25 flasks as adherent MDM cultures in RPMI media supple- 


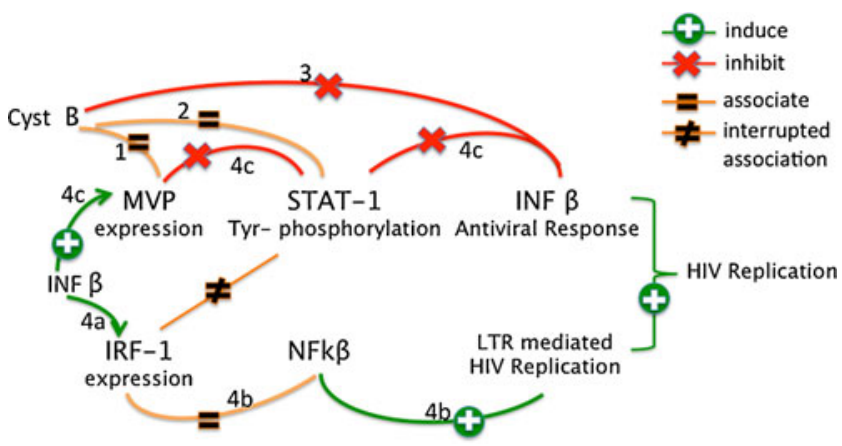

Fig. 6 Proposed mechanism for cystatin B stimulation of viral replication in macrophage reservoirs. Cystatin B may regulate STAT1 phosphorylation and thus control LTR-mediated replication and interferon-induced antiviral response, resulting in increased HIV replication in macrophage reservoirs. The numbers indicate the approach to be used to assess each step: (1) immunoprecipitation followed by LC-MS/MS, (2) immunoprecipitation followed by Western blot, (3) luciferase assay of IFN-treated and untreated Vero cells, (4) as reported in the literature: $4 a$ (Fujita et al. 1989); $4 b$ (Sgarbanti et al. 2008); 4c (Steiner et al. 2006)

mented with $10 \%$ human serum, $20 \%$ fetal bovine serum, $100 \mathrm{U} / \mathrm{mL}$ pen/strep, and $2 \mathrm{mM}$ glutamine. On day 7, MDMs were exposed to HIV- $1_{\mathrm{ADA}}$ stock using a multiplicity of infection of 0.1 by overnight incubation or left untreated as negative controls. One day after exposure to the virus, a full media exchange was performed; thereafter, a half media exchange was done every 2 days. Supernatants at 6,9 , and 12 days post-infection were collected to monitor infection by HIV-1 p24 antigen ELISA (Express BioTech International, Thurmont, MD) following the manufacturer's instructions. MDMs were maintained for 12 days in culture and lysed on ice with lysis buffer [ $5 \mathrm{mM}$ Tris- $\mathrm{HCl}$ buffer, $\mathrm{pH} 8,0.1 \%$ Triton X-100, and protease inhibitor cocktail (Sigma, St. Louis, MO)].

Immunoprecipitation Monoclonal antibodies against cystatin B (Sigma, St. Louis, MO) were used for protein-specific immunoprecipitation from cell lysates using an Exacta homologous IP/WB kit (Santa Cruz Biotechnology, Santa Cruz, CA) as described previously (Luciano-Montalvo and Melendez 2009). Whole cell lysates were pre-cleared by immunoprecipitation with beads for $1 \mathrm{~h}$, and the samples were incubated with antibody-conjugated beads overnight followed by several washes. The pre-cleared lysates were incubated with unconjugated beads as controls for nonspecific binding. The U87 whole cell lysates were used as a positive control for cystatin B.

$L C-M S / M S$ Immunoprecipitated proteins were digested in solution by an overnight incubation with trypsin $(0.1 \mu \mathrm{g} / \mu \mathrm{l})$ at $37^{\circ} \mathrm{C}$. Peptides were extracted $[60 \%$ acetonitrile with $0.1 \%$ trifluoroacetic acid (TFA)], dried, and then resuspended $(0.5 \%$ TFA) prior to purification using ZipTip ${ }^{\circledR}$ (Millipore Corpora- tion, Billerica, MA). Peptides were resuspended in $2 \%$ acetonitrile with $0.1 \%$ formic acid prior to fractionation on a microcapillary RP-C $\mathrm{C}_{18}$ column (New Objectives, Woburn, MA). The peptides were then fragmented and sequenced with a nanospray ionization configuration using a LTQ XL MS/MS (ThermoElectron, Thermo Fisher Scientific, Walthan, MA). The spectra obtained were searched against UniProtKB/Swiss-prot.fasta indexed for human proteins using the Turbo SEQUEST search engine (BioWorks 3.2). Protein hits were subjected to exclusion criteria and accepted as positive with high confidence if two or more unique peptides were sequenced per protein. Immunoprecipitation and protein identification experiments were performed with two different cultures from different donors.

Western blot analyses The MDM-immunoprecipitated proteins and Vero cell lysates were diluted with Laemmli sample buffer (BioRad, Hercules, CA) applied to a $10 \%$ Tris- $\mathrm{HCl}$ Ready Gel (BioRad, Hercules, CA) and transferred to 0.45$\mu \mathrm{m}$ nitrocellulose membranes (BioRad, Hercules, CA). After blocking with $5 \%$ bovine serum albumin in Tris-buffered saline, membranes were incubated with mouse anti-cystatin B (1:500, Sigma, St. Louis, MO). MDM-immunoprecipitated proteins were immunoblotted with mouse anti PKM2 (1:500, Abcam, Cambridge, MA) and rabbit anti-MVP (1:100, Abcam, Cambridge, MA). For immunoprecipitated proteins, the U87 and HeLa cell lysates were used as positive control for cystatin B. The Vero cells were immunoblotted with mouse anti-STAT-1PY (1:500 Abcam, Cambridge, MA). Empty vector was used as mock-transfected control and untransfected cells as negative controls. Mouse GAPDH $(1: 1,000)$ (Abcam) was used as the loading control. Signal was detected using the Super Signal ${ }^{\circledR}$ West Femto Maximum Sensitivity Substrate (Thermo). The density of protein bands was determined using the VersaDoc Imaging System (BioRad Laboratories, Hercules, CA) with Quantity One software and normalized against GAPDH.

Immunofluorescence and confocal imaging of MDM Monocyte isolation, cultivation, and HIV-1 infection were performed using the Lab-Tek Chamber Slides (Fisher, Pittsburgh, PA) in adherent cultures. The MDMs were fixed, permeabilized, and incubated overnight with anti-rabbit MVP (1:100, Abcam, Cambridge, MA) and anti-mouse STAT-1 (1:500, Abcam, Cambridge, MA) primary antibodies, and for $1 \mathrm{~h}$ with ALEXA-conjugated secondary antibody (Invitrogen, Carlsbad, CA). Confocal images were obtained on a Zeiss LSM 5 confocal laser-scanning microscope.

Co-transfection and luciferase reporter gene assays Vero cells, which are IFN deficient, were grown in minimum essential medium (Invitrogen, Carlsbad, CA) containing $10 \%$ fetal bovine serum to reach $70-80 \%$ confluence. To 
induce the expression of cystatin $\mathrm{B}, 7.5 \times 10^{5}$ cells were seeded in 6-well plates and incubated overnight. Three grams of Homo sapiens cystatin B as transfection-ready DNA NM_000100.2 inserted in pCMV6-XL5 (OriGene, Rockville, MD) or empty pcDNA3.1 vector was transfected with Lipofectamine (Invitrogen, Carlsbad, CA) according to the manufacturer's instructions, together with $1 \mathrm{~g}$ of a plasmid construct containing the ISG54 promoter fused to firefly luciferase and the SV40 promoter fused to Renilla luciferase (provided by Dr. Muñoz). The cells were stimulated with $500 \mathrm{U} / \mathrm{mL}$ of IFN- $\beta$ (PBL Interferon Source, Piscataway, NJ) in Opti-MEM I Reduced Serum Medium (Invitrogen, Carlsbad, CA) or left untreated. Twenty-four hours posttreatment, the cells were lysed and the luciferase activity was measured via a Triathler luminometer by using a dual luciferase assay (Promega) as recommended by the manufacturer. Firefly luciferase units were divided by Renilla units to obtain relative luciferase units.

Immunofluorescence and confocal imaging of Vero Cells Vero cells were grown, co-transfected, and stimulated with $500 \mathrm{U} / \mathrm{mL}$ of IFN- $\beta$ as described before. The Vero cells were fixed, permeabilized, and incubated overnight with anti-rabbit STAT-1 (1:500, Abcam, Cambridge, MA) primary antibodies and for $1 \mathrm{~h}$ with ALEXA-conjugated secondary antibody (Invitrogen, Carlsbad, CA). Bluefluorescent DAPI nucleic acid stain was used as the nuclear stain. Confocal images were obtained on a Zeiss confocal microscope Axiovert 200M with a LSM 510.

Acknowledgments We thank Dr. Howard Gendelman for providing HIV-1-ADA. We also thank Dr. Jorge Muñoz and Dr. Freddy Medina for giving us valuable suggestions and providing us the plasmid construct used for the luciferase assays. This work was supported by NIH grants R01 MH083516-01 and NCRR-RCMI G12 RR-03051 for the Translational Proteomics Center (to L.M.M.).

Open Access This article is distributed under the terms of the Creative Commons Attribution Noncommercial License which permits any noncommercial use, distribution, and reproduction in any medium, provided the original author(s) and source are credited.

\section{References}

Berger W, Steiner E, Grusch M, Elbling L, Micksche M (2009) Vaults and the major vault protein: novel roles in signal pathway regulation and immunity. Cell Mol Life Sci 66:43-61

Chang MA, Pine R, Klotman ME, Moore JP (2002) A soluble factor (s) secreted from CD8 T lymphocytes inhibits human immunodeficiency virus type 1 replication through STAT1 activation. J Virol 76:569-581

Chaudhuri A, Yang B, Gendelman HE, Persidsky Y, Kanmogne GD (2008) STAT1 signaling modulates HIV-1-1-induced inflammatory responses and leukocyte transmigration across the bloodbrain barrier. Blood 111:2062-2072
Christofk HR, Vander Heiden MG, Wu N, Asara JM, Cantley LC (2008) Pyruvate kinase M2 is a phosphotyrosine-binding protein. Nature 452:181-186

Ciborowski P, Kadiu I, Rozek W, Smith L, Bernhardt K, Fladseth M, Ricardo-Dukelow M, Gendelman HE (2007) Investigating the human immunodeficiency virus type 1-infected monocytederived macrophage secretome. Virology 363:198-209

Di Giamo R, Riccio M, Santi S, Galeotty C, Ambroosetti DC, Melli M (2002) New insights into molecular basis of progressive myoclonus epilepsy: a multiprotein complex with cystatin B. Hum Mol Genet 11:2942-2950

Fujita T, Reis LF, Watanabe N, Kimura Y, Taniguchi T, Vilcek J (1989) Induction of the transcription factor IRF-1 and interferonbeta mRNAs by cytokines and activators of second-messenger pathways. Proc Natl Acad Sci USA 86:9936-9940

Garcias-Crespo K, Cadilla C, Skolasky R, Meléndez LM (2009) Restricted HIV-1 replication in placental macrophages is caused by inefficient viral transcription. J Leukoc Biol 87:633-636

Gessani S, Puddu P, Varano B, Borghi P, Conti L, Fantuzzi L, Belardelli F (1994a) Induction of beta interferon by human immunodeficiency virus type 1 and its gp120 protein in human monocytes-macrophages: role of beta interferon in restriction of virus replication. J Virol 68:1983-1986

Gessani S, Puddu P, Varano B, Borghi P, Conti L, Fantuzzi L, Gherardi G, Bellardelli F (1994b) Role of endogenous interferonbeta in the restriction of HIV replication in human monocyte/ macrophages. J Leukoc Biol 56:358-361

Kinoshita S, Su L, Amano M, Timmerman LA, Kaneshima H, Nolan GP (1997) The T cell activation factor NF-ATc positively regulates HIV-1 replication and gene expression in $\mathrm{T}$ cells. Immunity 6:235-244

Kitazono M, Sumizawa T, Takebayashi Y, Chen ZS, Furukawa T, Nagayama S, Tani A, Takao S, Aikou T, Akiyama S (1999) Multidrug resistance and the lung resistance-related protein in human colon carcinoma SW-620 cells. J Natl Cancer Inst 91:1647-1653

Kitazono M, Okumura H, Ikeda R, Sumizawa T, Furukawa T, Nagayama S, Seto K, Aikou T, Akiyama S (2001) Reversal of LRP-associated drug resistance in colon carcinoma SW-620 cells. Int J Cancer 91:126-131

Kolli S, Zito CI, Mossink MH, Wiemer EA, Bennett AM (2004) The major vault protein is a novel substrate for the tyrosine phosphatase SHP-2 and scaffold protein in epidermal growth factor signaling. J Biol Chem 279:29374-29385

Luciano-Montalvo C, Meléndez L (2009) Cystatin B associates with signal transducer and activator of transcription 1 in monocytederived and placental macrophages. Placenta 30:464-467

Luciano-Montalvo C, Ciborowski P, Duan F, Gendelman H, Meléndez L (2008) Proteomic analyses associate cystatin B with restricted HIV-1-1 replication in placental macrophages. Placenta 29:10161023

Magnani M, Balestra E, Fraternale A, Aquaro S, Paiardini M (2003) Drug-loaded red blood cell-mediated clearance of HIV-1 macrophage reservoir by selective inhibition of STAT1 expression. J Leukoc Biol 74:764-771

Pitroda SP, Wakim BT, Sood RF, Beveridge MG, Beckett MA, MacDermed DM, Weichselbaum RR, Khodarev NN (2009) STAT1-dependent expression of energy metabolic pathways links tumour growth and radioresistance to the Warburg effect. BMC Med 7:68-78

Reynolds JL, Mahajan SD, Bindukumar B, Sykes D, Schwartz SA, Nair MP (2006) Proteomic analysis of the effects of cocaine on the enhancement of HIV-1-1 replication in normal human astrocytes (NHA). Brain Res 1123:226-236

Rodriguez JJ, Parisien JP, Horvath CM (2002) Nipah virus V protein evades alpha and gamma interferons by preventing STAT1 and 
STAT2 activation and nuclear accumulation. J Virol 76:1147611483

Rodriguez E, Plaud M, Romeu R, Skolasky R, Melendez L (2010) Late HIV-1 infection modulates the expression and activity of Cathepsin B, and its inhibitors in macrophages: implications in neuropathogenesis. Retrovirology 7:10

Sgarbanti M, Remoli AL, Marsili G, Ridolfi B, Borsetti A, Perrotti E, Orsatti R, Ilari R, Sernicola L, Stellacci E, Ensoli B, Battistini A (2008) IRF-1 is required for full NF-kappaB transcriptional activity at the human immunodeficiency virus type 1 long terminal repeat enhancer. J Virol 82:3632-3641

Steiner E, Holzmann K, Pirker C, Elbling L, Micksche M, Sutterlty H, Berger W (2006) The major vault protein is responsive to and interferes with interferon mediated STAT1 signals. J Cell Sci 119:459-469
Wang T, Gong N, Liu J, Kadiu I, Fraft-Terry SD, Schlautman JD, Ciborowski P, Volsky DJ, Gendelman HE (2008) HIV-1-infected astrocytes and the microglial proteome. J Neuroimmune Pharmacol 3:173-186

Wei L, Sandbulte MR, Thomas PG, Webby RJ, Homayouni R, Pfeffer LM (2006) NFkappaB negatively regulates interferon-induced gene expression and anti-influenza activity. J Biol Chem 281:11678-11684

Yi C, Li S, Chen X, Wiemer EA, Wang J, Wei N, Deng XW (2005) Major vault protein, in concert with constitutively photomorphogenic 1 , negatively regulates c-Jun-mediated activator protein 1 transcription in mammalian cells. Cancer Res 65:5835-5840

Yu Z, Fotouhi-Ardakani N, Wu L, Maoui M, Wang S, Banville D, Shen SH (2002) PTEN associates with the vault particles in HeLa cells. J Biol Chem 277(43):40247-40252 